\title{
AVALIAÇÃO NUTRICIONAL SUBJETIVA GLOBAL: INSTRUMENTOS PARA TRIAGEM EM CRIANÇAS HOSPITALIZADAS
}

\author{
NUTRITIONAL GLOBAL SUBJECTIVE EVALUATION: INSTRUMENTS \\ FOR SCREENING FOR HOSPITALIZED CHILDREN
}

\section{Leorenia Alves Bousquet ${ }^{a}$, Maria Luiza Ferreira Stringhini ${ }^{b}$, Andrea Sugai Mortoza ${ }^{c}$}

aleorenia@hotmail.com, bmluizastring@uol.com.br, candreasugai@gmail.com

Universidade Federal de Goiás - Goiânia (GO), Brasil

Data de recebimento do artigo: 21/07/2015

Data de aceite do artigo: 02/10/2015

\section{RESUMO}

Introduçáo: Ao longo dos últimos anos, diversas ferramentas de triagem nutricional têm sido desenvolvidas para aplicação no ambiente hospitalar. Estas possuem, como principal objetivo, a identificação precoce do risco nutricional, o que permite a avaliação e o diagnóstico nutricional prévio possibilitando ao profissional intervir antes do agravamento da desnutrição, prevenindo assim as suas consequências. A seleção de uma ferramenta uniforme e validada é uma questão importante na realização da triagem nutricional. Objetivos: Apresentar quatro métodos de triagem nutricional em pediatria e discutir suas vantagens e desvantagens. Metodologia: Levantamento de artigos científicos publicados nas bases de dados SciElo, LILACS, PubMed e MedLine no período de 1995 a 2015, nos idiomas inglês, português e espanhol. Foram utilizados os descritores "nutritional screening", "nutritional risk", "nutritional assessment" e "children". Foram incluídos estudos referentes a ferramentas de triagem nutricional desenvolvidas para crianças e adolescentes, e excluídos aqueles concernentes a adultos e idosos ou que traziam ferramentas criadas para doenças específicas. Resultado: Das quatro ferramentas de triagem nutricional estudadas, o Screening Tool for Risk of Impaired Nutritional Status and Growth (STRONGkids) foi apresentada como a ferramenta mais prática e de fácil utilizaçáo se comparada as outras, podendo ser aplicada por qualquer profissional da saúde no momento da admissão do paciente. Conclusáo: Com base nas características de cada método de triagem estudados, não se pôde eleger o mais adequado uma vez que todos apresentam limitaçôes na sua aplicação.

Palavras-chave: Triagem; estado nutricional; desnutrição; hospitalização; criança.

\section{ABSTRACT}

Introduction: Over the past few years, several nutritional screening tools have been developed for applicaa tion in hospitals. Those have, as main objective, the early identification of nutritional risk, which allow the evaluation and previous nutritional diagnosis enabling the professional to intervene before the worsening of malnutrition, preventing its consequences. The choice of a uniform and validated tool is an important issue in the execution of nutritional screening. Objectives: To present four methods of nutritional screening in pediatrics and discuss their advantages and disadvantages. Methodology: Survey of scientific articles published in databases like SciElo, LILACS, PubMed and MedLine from 1995 to 2015, in English, Portuguese and Spanish. The keywords used were: "nutritional screening", "nutritional risk", "nutritional assessment" and "children". Studies on nutritional screening tools developed for children and teenagers were included and the studies concerning adults and elderly were excluded. Those which dealt with tools designed for specific diseases were also excluded. Result: Of the four nutritional screening tools studied, the Screening Tool for Risk of Impaired Nutritional Status and Growth (STRONGkids) was presented as the easiest and most practical tool to use compared to others, and may be applied by any health professional in the moment of the patient's admission. Conclusion: Based on the characteristics of each screening method studied might not elect the most suitable because all of them have limitations in their application.

Keywords: Triage; nutritional status; malnutrition; hospitalization; child. 


\section{Introdução}

Desnutrição e risco nutricional são problemas comuns em pacientes hospitalizados. Estudos mundiais apontam que 20 a $50 \%$ dos pacientes adultos e pediátricos são desnutridos, sendo que alguns já são admitidos com desnutrição e outros a desenvolvem após a internaçãoo ${ }^{1-4}$.

No Brasil, o Inquérito Brasileiro de Avaliação Nutricional Hospitalar (IBRANUTRI), realizado pela Sociedade Brasileira de Nutrição Parenteral e Enteral, relatou que $48,1 \%$ dos pacientes hospitalizados estavam desnutridos, sendo que $12,6 \%$ desse grupo possuíam o diagnóstico de desnutrição grave e $35,5 \%$ de desnutrição moderada. Houve também progressão da desnutriçáo durante a internação, chegando a $61 \%$ quando a permanência no hospital foi maior que 15 dias $^{5}$.

Em crianças, esse problema continua a ser uma das principais causas de morbidade e mortalidade em todo o mundo e, no Brasil, o percentual de óbitos por desnutrição hospitalar grave se mantém em torno de $20 \%$, bem acima do limite de tolerância de 5\% estabelecido pela Organização Mundial de Saúde ${ }^{6}$. Esses óbitos geralmente ocorrem nas primeiras 48 horas de internação, mostrando as falhas do atendimento hospitalar a essas crianças ${ }^{7}$.

Vários fatores de risco estáo associados a essa síndrome carencial, como o baixo nível socioeconômico, presença de doenças prévias, perda de apetite, má absorção e aumento das necessidades energéticas ${ }^{8,9}$. Em crianças hospitalizadas se destacam as seguintes condiçóes relacionadas à desnutrição: idade inferior a seis meses, baixo peso ao nascimento, história de prematuridade, baixo grau de escolaridade materna, renda familiar abaixo de dois salários mínimos, saneamento básico domiciliar inadequado e história de diarreia prévia ${ }^{5,10}$.

A desnutrição contribui adversamente à evolução clínica de pacientes hospitalizados e os resultados são aumento ou risco de infecçóes, atraso na cicatrização de feridas, edemas por hipoproteinemia, diminuição da motilidade intestinal, tendência ao choque e supressão imunológica ${ }^{11,4}$. O déficit nutricional também é fortemente correlacionado com o aumento da morbimortalidade, tempo de internação prolongado e aumento dos custos hospitalares, já que o custo para tratar pacientes desnutridos é quatro vezes maior do que o necessário para tratar o bem nutrido ${ }^{4,11-13}$.

Ao longo dos últimos anos, diversas ferramentas de triagem nutricional para aplicação no ambiente hospitalar têm sido desenvolvidas. O principal objetivo destas é a identificação precoce do risco nutricional, o que permite a avaliação e o diagnóstico nutricional prévio, possibilitando ao profissional intervir antes do agravamento da desnutrição, prevenindo assim as suas consequências ${ }^{1,14}$.

Entretanto, no Brasil, não há técnica padrão de triagem nutricional. Assim, é necessário um senso crítico para a escolha da ferramenta mais indicada ao ambiente hospitalar - já que não há consenso sobre o melhor instrumento de triagem nutricional, uma vez que os descritos na literatura apresentam limitaçóes, vantagens e desvantagens quando utilizadas em populaçóes específi$\operatorname{cas}^{1}$. Ressalta-se que estudos referentes à avaliação nutricional em pediatria são escassos, sendo mais estabelecida a importância dos métodos baseados em adultos ${ }^{7,15-17}$.

Para realizar a triagem nutricional adequada, a seleção de uma ferramenta uniforme e validada é claramente uma questão importante. Diante disso, o objetivo desta revisão é apresentar quatro métodos de triagem nutricional em pediatria (Pediatric Nutritional Risk Score; Subjecive Global Nutrition Assessment, Screening Tool for the Assessment of Malnutrition in Pediatrics; Screening Tool for Risk of Impaired Nutritional Status and GrowthSTRONGkids) e discutir vantagens e desvantagens.

\section{Método}

A fundamentação teórica foi realizada por meio de levantamento bibliográfico de artigos científicos publicados nas bases de dados virtuais - Scientific Electronic Library (SciELO), Latin American and Caribbean Center on Health (LILACS), United States National Library of Medicine (PubMed) e Medical Literature Analysis and Retrieval System Online (MedLine) - e em livros técnicos, entre setembro e outubro de 2014. A busca foi definida pela combinação das seguintes palavras-chave em português, inglês e espanhol: nutritional screening, nutritional risk, nutritional assessment e children.

Duzentos e oitenta e dois estudos sobre o tema publicados nos últimos 20 anos foram resgatados. A seleção das publicações foi baseada nos títulos e resumos, resultando em 30 estudos. Destes, dez foram incluídos neste trabalho, dos quais nove foram trabalhos originais e um de revisão, além de dois livros técnicos. Foram selecionadas as quatro ferramentas de triagem nutricional mais conhecidas, disponíveis na literatura e, após obtenção dos artigos completos sobre as ferramentas de triagem nutricional, cada uma foi revisada e sua descrição realizada. Estudos referentes a ferramentas de triagem nutricional desenvolvidas para crianças e adolescentes foram incluídos, e excluídos aqueles concernentes a adultos e idosos ou, que traziam ferramentas criadas para doenças específicas.

\section{Risco e triagem nutricional}

$\mathrm{O}$ risco nutricional se refere ao perigo aumentado de morbimortalidade em decorrência do estado nutricional, sendo um fator preditor de complicaçóes em pacientes hospitalizados. É avaliado combinando-se estado nutricional atual e a gravidade da doença, utilizando-se dados objetivos como: altura, peso corporal, alteração de peso, diagnóstico e presença de comorbidades ${ }^{1,18}$. 
O reconhecimento do risco nutricional se faz por meio da triagem nutricional, definida como processo de identificação das características melhor associadas a problemas dietéticos ou nutricionais ${ }^{19}$. É aplicada por meio de inquéritos simples ao paciente ou a seus familiares, que são mais preditivos de desnutriçáo, constatando mudanças na condição clínica que afetem o estado nutricional do indivíduo ${ }^{1,19,20}$.

$\mathrm{Na}$ ausência de procedimentos de triagem formais, mais da metade dos pacientes com risco de desnutriçáo não são identificados e/ou encaminhados para tratamento. A falta de uma ferramenta de triagem nutricional amplamente aceita para a detecção de pacientes em risco de desnutrição é frequentemente vista como um fator que dificulta tanto o reconhecimento quanto a eficácia do tratamento ${ }^{20}$.

Em 2005, o Ministério da Saúde brasileiro (MS), por meio do reconhecimento da importância do rastreamento do risco de desnutrição, tornou obrigatória a implantação de protocolos de triagem e avaliação nutricional em hospitais do Sistema Único de Saúde (SUS). Ainda segundo o MS, se identificado risco nutricional, uma avaliação completa do estado nutricional, seguido de planejamento dietético deve ser estabelecido pela equipe de saúde ${ }^{21}$.

\section{Métodos de triagem ou de risco nutricional em pediatria}

As principais ferramentas de triagem nutricional validadas e utilizadas em pediatria são: Pediatric Nutritional Risk Score (PNRS), Subjective Global Nutrition Assesment (SGNA), Screening Tool for the Assessment of Malnutrition in Pediatrics (STAMP) e STRONGkids. Para maior compreensão dos estudos, essas ferramentas serão descritas a seguir (Tabela 1).

Tabela 1: Métodos de triagem nutricional estudados e suas variáveis.

\begin{tabular}{|c|c|c|c|c|}
\hline Variáveis & PNRS & SGNA & STAMP & STRONGkids \\
\hline Doença & X & & X & $\mathrm{X}$ \\
\hline Dor & $\mathrm{X}$ & & & \\
\hline Ingestão dietética & $\mathrm{X}$ & $\mathrm{X}$ & $\mathrm{X}$ & $\mathrm{X}$ \\
\hline Peso e altura & & $\mathrm{X}$ & $\mathrm{X}$ & \\
\hline $\begin{array}{l}\text { Adequação de } \\
\text { peso/altura }\end{array}$ & & $\mathrm{X}$ & & \\
\hline $\begin{array}{l}\text { Perda ponderal } \\
\text { indesejada }\end{array}$ & & $\mathrm{X}$ & & $\mathrm{X}$ \\
\hline $\begin{array}{l}\text { Sintomas } \\
\text { gastrointestinais }\end{array}$ & & $\mathrm{X}$ & & \\
\hline $\begin{array}{l}\text { Capacidade } \\
\text { funcional }\end{array}$ & & $\mathrm{X}$ & & \\
\hline Fator de estresse & & $\mathrm{X}$ & & \\
\hline Exame físico & & $\mathrm{X}$ & & $\mathrm{X}$ \\
\hline
\end{tabular}

PNRS = Pediatric Nutritional Risk Score; SGNA = Subjective Global Nutritional Assessment; STAMP $=$ Screening Tool for the Assessment of Malnutrition in Pediatrics; STRONGkids $=$ Screening Tool for Risk of Impaired Nutritional Status and Grow.

\section{Escore do risco nutricional pediátrico ou Pediatric Nutricional Risk Score (PNRS)}

Esta ferramenta de triagem nutricional foi desenvolvida em um hospital situado em Paris, com 26 crianças entre zero e 17 anos. Sáo observadas, nas primeiras 48 horas após admissão hospitalar, (1) a ingestão alimentar, (2) a condição patológica (classificada em leve, moderada e severa) e (3) a presença de sintomas como dor (verificada pelo choro constante, movimentos anormais e outros comportamentos), dispneia e depressão. Também consta desta ferramenta um recordatório alimentar, a fim de estimar se a ingestão alimentar se encontra maior ou menor que $50 \%$ das necessidades estimadas. ${ }^{22}$

\section{Avaliação nutricional subjetiva global ou Subjective Global Nutrition Assesment (SGNA)}

A avaliação nutricional subjetiva global é uma adaptação da ferramenta avaliação subjetiva global (SGA) utilizada em adultos. Sua criação se deu mediante a necessidade de identificação do risco nutricional pré e pós-cirúrgico em pacientes pediátricos com idade superior a um mês e inferior a 18 anos. Foram estudadas 175 crianças admitidas para grandes cirurgias torácicas e cardíacas em um hospital geral de Toronto, Canadá ${ }^{3}$.

No questionário são consideradas informaçôes recentes da criança como: (1) altura e peso (da criança e dos pais); (2) ingestão alimentar (tipo, volume e frequências das mamadas e sólidos para bebês e frequência de alimentação com uma breve descrição da ingestão de um dia típico para crianças, classificação do apetite e alteraçôes recentes, problemas com a alimentação e restriçōes dietéticas); (3) sintomas gastrointestinais (perda do apetite, náuseas, vômitos, diarreia e constipação para todas as idades e dor de estômago e náuseas para crianças); (4) Mudanças recentes na capacidade funcional (estado de alerta para bebês e frequência escolar, capacidade de correr e brincar com jogos ou esportes e tempo de sono para crianças); (5) Exame físico para detecção de perda muscular e gordura subcutânea, assim como edema. Após avaliação subjetiva, classifica-se a criança em três grupos: bem nutrida, moderadamente mal nutrida ou severamente mal nutrida ${ }^{23}$.

\section{Screening Tool for the Assessment of Malnu- trition in Pediatrics (STAMP)}

A ferramenta STAMP foi desenvolvida por pesquisadores britânicos para avaliação de crianças e adolescentes entre dois e 17 anos. Foram estudadas 
110 crianças e considerou-se para avaliação de desnutrição em pediatria três elementos: diagnóstico clínico em que se avaliam doenças que tenham implicação nutricional; ingestão nutricional; avaliação antropométrica com enfoque no peso e altura do paciente, sendo necessária a utilização de gráfico de crescimento ou tabela de percentil para análise das informaçôes coletadas. Cada elemento é, portanto, pontuado, sendo considerado em risco nutricional pontuação maior que três. Após classificação do risco, indica-se o desenvolvimento de um plano de cuidado nutricional sendo uma meta estabelecida baseada no risco de desnutrição encontrado na criança. ${ }^{24}$

\section{Screening Tool for Risk of Impaired Nutritio- nal Status and Growth (STRONGkids)}

A ferramenta STRONGkids foi desenvolvida por pesquisadores holandeses em 423 pacientes de um mês a 18 anos, internados em 44 hospitais. Essa ferramenta é composta pelas etapas: (1) avaliaçấo subjetiva global em que se avalia a perda de massa muscular e adiposa; (2) alto risco da doença, a qual indica também o risco ou previsão de uma cirurgia de grande porte; (3) ingestáo e perdas nutricionais (diminuiçáo da ingestáo alimentar e presença de diarreia e vômito); (4) dificuldade de ganho de peso ou perda de peso. A cada item é dada uma pontuação, fornecida quando a resposta à pergunta for positiva. A somatória desses pontos identifica o risco de desnutrição permitindo ao aplicador decidir sobre a intervenção e acompanhamento necessário ${ }^{25}$.

\section{Discussão}

$\mathrm{Na}$ busca de uma ferramenta de triagem nutricional, deve-se eleger a mais completa possível e ao mesmo tempo, a de melhor aplicabilidade ao serviço ou à clientela com que se trabalha. Especificaçóes como o maior número de profissionais que podem aplicá-la, tempo de duração da aplicação, utilização ou não de recursos financeiros e capacidade de detectar o risco nutricional com confiança devem ser levados em consideraçăo ${ }^{1,18}$.

Em 2003, a Sociedade Europeia de Nutrição e Metabolismo (ESPEN) declarou os princípios que devem constar nas ferramentas de triagem nutricional ${ }^{26}$ : (1) Qual é o estado nutricional atual do paciente? Este item refere-se à composição corporal atual do paciente. Altura e peso podem ser aferidos para permitir o cálculo de escores ou IMC; ${ }^{(2)} \mathrm{A}$ condição é estável? Este item aborda recentes perdas de peso do paciente, dados esses que podem ser obtidos a partir do histórico do paciente ou a partir de mediçóes anteriores em registros médicos; ${ }^{(3)}$ Será que a condiçãa nutricional atual pode piorar? Essa pergunta pode ser respondida perguntando se a ingestão de alimentos foi reduzida até o momento da triagem e se sim, por quanto tempo; ${ }^{(4)}$ Será que o processo da doença acelera a deterioraçáo nutricional? Esse item aborda o processo da doença subjacente, que pode aumentar as necessidades nutricionais devido ao estresse metabólico associado à gravidade da doença de base (por exemplo, grande cirurgia, sepse e múltiplos traumas), fazendo que o estado nutricional se agrave mais rapidamente.

Desses princípios, as variáveis de 1 a 3 devem estar incluídas em qualquer ferramenta de rastreio nutricional, sendo a quarta variável relevante em ambientes hospitalares. Para cada variável deve ser atribuída uma pontuação, quantificando assim, o grau de risco nutricional ou de desnutrição, permitindo traçar uma meta de ação ${ }^{26}$.

Em relação a tais princípios, as ferramentas SGNA e STRONGkids incorporam todos os itens em suas ferramentas, não utilizando medidas antropométricas para definição do estado nutricional e sim avaliação clínica subjetiva $^{23,25}$. Os instrumentos SGNA e PNRS incluem itens adicionais (como sintomas gastrointestinais, capacidade funcional e altura dos pais) para SGNA e dor para PNRS 22,23 .

Quanto à eficiência das ferramentas estudadas, a reprodutibilidade (confiabilidade) foi testada nas ferramentas SGNA e STAMP, por um grupo de nutricionistas, mostrando concordância em ambas ${ }^{23,24}$. Sensibilidade, especificidade e validade preditiva foram testadas na ferramenta STAMP, sendo a avaliação dietética completa utilizada como padrão-ouro para tal julgamento. Os resultados mostraram que esse instrumento apresenta uma sensibilidade de $72 \%$, especificidade de $90 \%$ e validade preditiva de $55 \%{ }^{24}$.

A aplicabilidade (ou seja, a facilidade e o tempo de aplicação da ferramenta) só foi descrita no trabalho original da PNRS, que despendeu 48 horas para completar os itens da ferramenta ${ }^{22}$. As outras ferramentas fazem apenas menção de facilidade e rapidez de utilização, possibilitando ao avaliador concluir a triagem na admissão ${ }^{23-25}$.

Moeeni et al. ${ }^{27}$ avaliaram a facilidade de aplicação da STRONGkids, quando utilizada por enfermeiros e pediatras. Das 162 crianças do estudo, 11,7\% estavam desnutridas. Destas, a ferramenta STRONGkids classificou $84 \%$ como em alto risco, quando aplicada por enfermeiros, e 90\%, quando aplicada por pediatras. Em conclusão, houve concordância (Kappa $=0,65$ ) entre os resultados, demonstrando a facilidade da utilização dessa ferramenta entre profissionais de diferentes categorias.

Em um estudo transversal ${ }^{28}$, comparando a ferramenta STRONGkids e STAMP, obteve-se um tempo médio de aplicação da primeira de cinco minutos e, da 
segunda, de 10 a 15 minutos. Quanto a viabilidade da ferramenta, foi descrita apenas no manuscrito original da STRONGkids ${ }^{25}$, podendo o instrumento ser aplicado em $98 \%$ dos indivíduos do estudo.

As ferramentas de triagem nutricional, com exceção da SGNA, trazem um sistema de pontuação, que variam de zero a dois para cada elemento, sendo o número total de pontos o preditor do grau de risco nutricional do paciente. De acordo com os pontos obtidos, o paciente é classificado em baixo, moderado e alto risco de desnutrição para PNRS, STRONGkids e STAMP, e bem nutrido, moderamente mal nutrido ou severamente mal nutrido para SGNA. Entretanto, os escores totais máximos e pontos de corte para a classificaçáo dos grupos de risco são diferentes dentro de cada ferramenta ${ }^{22-25}$.

Essa variação no sistema de pontuação traz diferenças consideráveis na porcentagem de pacientes em cada grupo de risco nos estudos originais, nos quais a quantidade de pacientes considerados de alto risco foi de $45 \%$, 9\%, 15\% e $18 \%$ para PNRS, STRONGkids, SGNA e STAMP, respectivamente ${ }^{22-25}$. Moderado risco foram de $41 \%, 53 \%$ e $36 \%$ para PNRS, STRONGkids e STAMP, respectivamente ${ }^{22,23,25}$.

Diferentes estudos (Tabela 2) trazem a tentativa de comparação entre as ferramentas de triagem nutricional em pediatria. Moeeni et al. ${ }^{29}$ realizaram um estudo em um hospital iraniano com 150 crianças comparando STRONGkids, STAMP e Paediatric Yorkhill Malnutrition Score com medidas antropométricas, sendo que a STRONGkids obteve maior correlação com as medidas antropométricas que as demais ferramentas. Além disso, na estratificação de risco obtida pela STRONGkids, observou-se relação direta maior com o tempo de internação hospitalar.

Tabela 2: Diferentes estudos, seus objetivos e resultados.

\begin{tabular}{|c|c|c|}
\hline Referência & Objetivo do estudo & Resultado \\
\hline Moeeni et al., 2014 & Confiabilidade da STRONGkids. & Ferramenta pode ser aplicada por diferentes profissionais. \\
\hline Ling et al., 2011 & $\begin{array}{l}\text { Tempo médio de aplicação } \\
\text { STRONGkids x STAMP. }\end{array}$ & $\begin{array}{l}\text { STRONGkids }=5 \mathrm{~min} \\
\text { STAMP }=10 \text { a } 15 \mathrm{~min}\end{array}$ \\
\hline Moeeni et al., 2012 & $\begin{array}{l}\text { STRONGkids e STAMP x } \\
\text { Medidas antropométricas. }\end{array}$ & $\begin{array}{l}\text { STRONGkids obteve maior correlação com as medidas antropomé- } \\
\text { tricas que STAMP. }\end{array}$ \\
\hline More et al., 2012 & $\begin{array}{l}\text { STAMP x Exame físico, antropo- } \\
\text { metria e composição corporal. }\end{array}$ & $\begin{array}{c}\text { Ao comparar com avaliçáo clínica, STAMP demonstrou sensibilida- } \\
\text { de de } 90 \% \text { na detecçáo de pacientes desnutridos. }\end{array}$ \\
\hline Wiskin et al., 2012 & $\begin{array}{l}\text { STAMP, STRONGkids, SGNA, } \\
\text { PNRS x Grau de desnutrição de } \\
\text { acordo com a OMS. }\end{array}$ & $\begin{array}{l}\text { O risco nutricional obtido pelas ferramentas de triagem nấo é con- } \\
\text { fiável, quando mensurados em crianças com doenças crônicas. }\end{array}$ \\
\hline Margineãn et al., 2014 & $\begin{array}{l}\text { STRONGkids } \mathrm{x} \text { escores de risco } \\
\text { da OMS. }\end{array}$ & $\begin{array}{l}\text { Relação significativa entre o risco de desnutrição determinado pela } \\
\text { STRONGkids e a presença de desnutriçáo aguda ou crônica (OMS). }\end{array}$ \\
\hline Durakbasa et al., 2014 & $\begin{array}{l}\text { STRONGkids } \mathrm{x} \text { escores de risco } \\
\text { da OMS }\end{array}$ & $\begin{array}{l}\text { Relação significativa entre o risco de desnutrição determinado pela } \\
\text { STRONGkids e a presença de desnutriçáo aguda ou crônica (OMS). }\end{array}$ \\
\hline Spagnuolo et al., 2013 & $\begin{array}{l}\text { STRONGkids } \mathrm{x} \text { medidas } \\
\quad \text { antropométricas. }\end{array}$ & $\begin{array}{c}\text { Confiabilidade e eficácia da STRONGkids é limitada, devido o } \\
\text { sistema de pontuação, que parece ser afetado pela alta pontuação } \\
\text { dada a doenças de base. }\end{array}$ \\
\hline Joosten et al., 2014 & $\begin{array}{l}\text { Consideraçôes metodológicas } \\
\text { sobre ferramentas de triagem em } \\
\text { crianças hospitalizadas. }\end{array}$ & STRONGkids é mais prática do que as outras ferramentas. \\
\hline
\end{tabular}

PNRS = Pediatric Nutritional Risk Score SGNA = Subjective Global Nutritional Assessment STAMP = Screening Tool for the Assessment of Malnutrition in Pediatrics; STRONGkids = Screening Tool for Risk of Impaired Nutritional Status and Growt.

More et al. $^{30}$, em um estudo transversal realizado na Espanha, compararam o questionário STAMP com exame físico, antropometria e composição corporal e, como resultado, observaram que $48,4 \%$ das crianças estavam em alto risco nutricional. Ao comparar esse resultado com a avaliação clínica, a ferramenta STAMP demonstrou uma sensibilidade de $90 \%$ na detecção de pacientes desnutridos.
Ling et $\mathrm{al}^{28}$, em um estudo realizado no Reino Unido, comparou STAMP com STRONGkids, em que dois pesquisadores treinados aplicaram as ferramentas em pacientes elegíveis. Como resultado, a STAMP classificou como alto risco $44 \%$, médio risco $28 \%$ e baixo risco $28 \%$, enquanto a STRONGkids classificou como alto risco $27 \%$, médio risco $49 \%$ e baixo risco $24 \%$. Assim, concluiu-se que STRONGkids foi superior a 
STAMP em termos de classificação de risco, já que a STAMP superestimou o número de crianças com alto risco nutricional.

Estudo realizado por Wiskin et al. ${ }^{31}$ em 46 crianças com doença inflamatória intestinal atendidas em ambulatório ou hospitalizadas comparou as ferramentas STAMP, STRONGkids, SGNA e PNRS com o grau de desnutrição obtido por dados antropométricos classificados pela Organizaçáo Mundial de Saúde. Como resultado, não houve concordância entre as diferentes categorias de risco estabelecidas pelas ferramentas e grau de desnutrição avaliado por medidas antropométricas. Os autores concluíram que o risco nutricional obtido pelas ferramentas de rastreio nutricional não é confiável, quando mensurado em crianças com doenças crônicas.

Dois estudos realizados com o objetivo de investigar a eficácia da ferramenta STRONGkids por meio de comparaçáo dos escores de risco com o estado nutricional real de crianças, trazem relação significativa entre o risco de desnutriçáo determinado por essa ferramenta e a presença de desnutrição aguda ou crônica ${ }^{32,33}$. Entretanto, Spagnuolo et al..$^{34}$, em um estudo observacional envolvendo 150 crianças em 12 hospitais italianos, mostraram $70 \%$ das crianças em risco moderado ou grave pela STRONGkids, porém, apenas $20 \%$ delas eram realmente desnutridas pelas medidas antropométricas. Os autores sugerem, que a confiabilidade e eficácia da STRONGkids é limitada, sendo o principal fator limitante o sistema de pontuação, que parece ser afetado pela alta pontuação dada a doença de base.

Além do objetivo principal de triar o paciente por meio da avaliação de risco nutricional, STAMP e SGNA também objetivam avaliar o estado nutricional do paciente na admissão ${ }^{22-25}$. Considera-se, então, importante fazer a distinção entre as ferramentas projetadas para avaliar o risco de se ficar desnutrido durante a internação, das ferramentas desenvolvidas para avaliar o estado nutricional real ou desnutrição na admissão ${ }^{35}$. Ressalta-se que pacientes cronicamente desnutridos e classificados como de alto risco na admissão necessitam de avaliação nutricional completa e intervenção nutricional imediata ${ }^{6}$.

A questão mais controvertida na validação de uma ferramenta de triagem nutricional é a possibilidade de prever o estado nutricional atual do paciente. A ferramenta STAMP, por exemplo, utiliza como padrão-ouro uma avaliação dietética completa. Contudo, questiona-se se tal método realmente seria padrão-ouro, já que nem todos os países possuem o profissional nutricionista para sua aplicação e o papel desse profissional pode variar de acordo com o país ${ }^{35}$.

Outro fator limitante é a inexistência de uma definição universalmente aceita para a desnutrição, que pode ser classificada de acordo com sua etiologia, quadro clínico, características morfológicas, antropométricas e outras $^{36}$. Diante disso, a antropometria, pela sua facilidade de execuçáo e baixo custo, se revela como método isolado mais utilizado (principalmente na infância) e assim, várias escalas podem ser usadas para se comparar as medidas antropométricas com um padrão de referência, sendo as mais comuns o percentil e o escore $\mathrm{z}^{37}$.

De acordo com a Organização Mundial da Saúde, uma pontuaçáo inferior à escore $\mathrm{z}$ - dois de peso para altura e altura para idade - é adotada para a desnutrição aguda e crônica, respectivamente ${ }^{36}$. Essa definição, quando aplicada para STRONGkids e SGNA, mostrou diferença significativa entre a média de desvio padrão e/ ou o número de crianças com desnutrição nas diferentes categorias de risco ${ }^{23,25}$.

Mesmo diante das limitações encontradas, Joosten et al. ${ }^{35}$ e Moeeni et al..$^{27,29}$ concluíram que a ferramenta STRONGkids se mostrou mais prática e de fácil utilização se comparada às outras, podendo ser aplicada por qualquer profissional da saúde diretamente na admissão do paciente. Tal conclusão se baseou em sua composição por quatro itens simples, de fácil entendimento, por dispensar a antropometria e permitir identificar risco no estado nutricional.

\section{Considerações finais}

Com base nas características de cada método estudado, não se pôde eleger a ferramenta mais adequada pois todas apresentam limitações. Na prática clínica, o instrumento de triagem ideal é aquele que pode, de forma rápida e confiável, triar o estado nutricional da criança, de modo a identificar os grupos de alto risco que necessitem de avaliação mais detalhada e intervenção nutricional urgente.

A adoção de instrumentos de triagem nutricional deve ser parte da atenção primária de todas as crianças internadas no hospital, onde técnicas de rastreamento nutricional e a sistematização de sua aplicação devem ser padronizadas, já que a detecção do risco de desnutrição hospitalar auxilia na adequação do tratamento nutricional, previne a instalação da desnutrição e melhora o prognóstico do paciente hospitalizado. Uma vez que o MS preconiza a triagem nutricional no âmbito hospitalar, a prática de triagem validada para a população infantil brasileira e sua aplicação ainda não é bem estabelecida ou não há ferramentas. Portanto, são necessários incentivos governamentais para a realização de práticas que possibilitem novos estudos.

\section{Referências}

1. Raslan M., Gonzales MC, Dias MCG, Paes-Barbosa FC, Cecconello I, Waitzberg DL. Aplicabilidade dos métodos 
de triagem nutricional no paciente hospitalizado. Rev Nutr 2008; 21(5):553-61.

2. Liang X, Jiang ZM, Noalan MT, Wu X, Zhang H, Zheng Y et al. Nutritional risk, malnutrition (undernutrition), overweight, obesity and nutrition support among hospitalized patients in Beijing teaching hospitals. Asia Pac J Clin Nutr 2009; 18(1):54-62.

3. Giryes S, Leibovitz E, Matas Z, Fridman S, Gavish D, Shalev B et al. MEasuring NUtrition Risk in Hospitalized Patients: MENU, A. Hospital-Based Prevalence Survey. Isr Med Assoc J 2012; 14(7):405-9.

4. Karateke F, Ikiz GZ, Kuvvetli A, Menekse E, Das K, Ozyazici $S$ et al. Evaluation of nutritional risk screening-2002 and subjective global assessment for general surgery patients: a prospective study. J Pak Med Assoc 2013; 63(11):1405-8.

5. Waitzberg DL, Caiaffa WT, Correia MI. Hospital malnutrition: The Brazilian national survey (IBRANUTRI): a study of 40000 patients. Nutrition 2001; 17 (7-8):573-80.

6. Brasil. Ministério da Saúde. Manual de atendimento da criança com desnutrição grave em nível hospitalar. Brasília: Ministério da Saúde; 2005.

7. Monte CMG, Sarni R. Tratamento hospitalar da criança gravemente desnutrida. Temas de Nutrição em Pediatria, [S.1.]. SBP 2002; 2(1):24-50.

8. Waitzberg DL, Correia MI. Nutritional assessment in the hospitalized patient. Am J Clin Nutr 2000; 6(5):531-8.

9. Correia MITD, Campos ACL. Prevalence of Hospital Malnutrition in Latin America: The Multicenter ELAN Study. Rev Nutr 2003; 19(1):823- 5.

10. Falbo AR, Bezerra AJG. Desnutrição grave: alguns aspectos clínicos e epidemiológicos de crianças hospitalizadas no Instituto materno infantil de Pernambuco (IMIP), Brasil. Cad Saúde Pública 2002; 18(1):1473-7.

11. Duchini L, Jordão AA, Brito TT, Diez-Garcia RW. Avaliação e monitoramento do estado nutricional de pacientes hospitalizados: uma proposta apoiada na opiniáo da comunidade científica. Rev Nutr 2010; 23(4):513-22.

12. Sorensen J, Kondrup J, Prokopowicz J, Schiesser M, Krăhenbuhl L, Meier R et al. EuroOOPS study group. EuroOOPS: An international, multicentre study to implement nutritional risk screening and evaluate clinical outcome. Clin Nutr 2008; 27(3):340-9.

13. Mello ED, Beghetto MG, Teixeira LB, Luft VC. A competência dos profissionais em identificar a desnutrição hospitalar. Rev Bras Nutr Clín 2003; 18(4):173-7.

14. Van Bokhorst de Van Der Schueren MA, Guaitoli PR, Jansma EP, VET HC. Nutrition screening tools: does one size fit all? A systematic review of screening tools for the hospital setting. Clin Nutri 2014; 33(1):39-58.

15. Ferreira HS, França AO. Evolução do estado nutricional de crianças submetidas à internação hospitalar. J Pediatr 2002; 1(78):491-6.

16. Monte CMG. Atendimento à criança desnutrida em ambulatório e comunidade. Temas de Nutrição em Pediatria,
[S.l.]: Departamento Cientifico de Nutrição da Sociedade Brasileira de Pediatria 2002; 2:13-23.

17. Medeiros AQ, Pinto ICS, SILVA CR. Avaliação Nutricional. In: Vasconcelos MJOB, Barbosa JM, Pinto ICS, Lima TM, Araujo AFC. Nutrição Clínica: Obstetrícia e Pediatria. Rio de Janeiro: MedBook; 2011:211-38.

18. Araujo MAR, Lima LS, Ornelas GC, Logrado MHG. Análise comparativa de diferentes métodos de triagem nutricional do paciente internado. Com Ciências Saúde 2010; 21(4):331-42.

19. Barrocas A. Rastreamento nutricional. In: Waitzberg DL. Nutrição oral, enteral e parenteral na prática clínica. $4^{\mathrm{a}} \mathrm{ed}$. São Paulo: Atheneu; 2009:303-21.

20. Neelemaat F, Meijers J, Kruizenga H, Van Ballegooijen H, Schueren MB. Comparison of five malnutrition-screening tools in one hospital inpatient sample. J Clin Nurs 2011; 20(1):2144-52.

21. Brasil - Diário Oficial da República Federativa do Brasil. Portaria Secretaria de Atenção à saúde no 131 , de 8 de março de 2005. Organização e implantação de Serviços de Assistência e Centros de Referência de Alta complexidade [no âmbito do Sistema Único de saúde]. Available from: http://dtr2001. saude.gov.br/sas/PORTARIAS/Port2005/PT-131.htm.

22. Sermet-Gaudelus I, Poisson-Salomon S, Colomb V, Brusset $\mathrm{MC}$, Mosser F, Berrier F et al. Simple pediatric nutritional risk score to identify children at risk of malnutrition. Am J Clin Nutr 2000; 72(1):64-70.

23. Secker DJ, Jeejeebhoy KN. Subjective Global Nutrition Assessment for children. Am J Clin Nutr 2007; 85(1):1083-9.

24. McCarthy H, Dixon M, Crabtree I, Eaton-Evanst MJ, McNulty $\mathrm{H}$. The development and evaluation of the Screening Tool for the Assessment of Malnutrition in Pediatrics (STAMPC) for use by healthcare staff. J Hum Nutr Diet 2012; 25(4):311-8.

25. Hulst JM, Zwart H, Hop WC, Joosten KFM. Dutch national survey to test the STRONGkids nutritional risk screening tool in hospitalized children. Clin Nutr 2010; 29:106-11.

26. KONDRUP J, ALLISON SP, ELIA M, VELLAS B, PLAUTH M. ESPEN guidelines for nutrition screening 2002. Clin Nutr 2003; 22(4):415-21.

27. Moeeni V, Wakks T, Day AS. The STRONGkids nutritional risk screening tool can be used by pediatrics nurses to identify hospitalized children at risk. Acta Paediatr 2014; 103(12):528-31.

28. Ling RE, Hedges V, Sullivan PB. Nutritional risk in hospitalised children: anassessment of two instruments. e-SPEN, Eur e-Journal Clin Nutr Metab 2011; 6:153-7.

29. Moeeni V, Wakks T, Day AS. Assessment of nutritional status and nutritional riskin hospitalized Iranian children. Acta Paediatr 2012; 101(10):446-51.

30. More LRA, López MA, Álvarez HM, Chicano CS, Martínez GR, Ruzafa LE et al. Validation of a nutritional screening 
tool for hospitalized pediatric patients. Nutr Hosp 2012; 27(5):1429-36.

31. Wiskin AE, Owens DR, Cornelius VR, Wootton SA, Beattie RM. Paediatricnutrition risk scores in clinical practice: children with inflammatory boweldisease. J Hum Nutr Diet 2012; 25(4):319-22.

32. Márginean $\mathrm{O}$, Pitea $\mathrm{AM}$, Voidãzan $\mathrm{S}$, Márginean $\mathrm{C}$. Prevalence and Assessment of Malnutrition Risk among Hospitalized Children in Romania. J Health Popul Nutr 2014; 32(1):97-102.

33. Durakbasa ÇU, Fettahoglu S, Bayar A, Mutus M, Okur $H$. The prevalence of Malnutrition and Effectiveness of STRONGkids Tool in the Identification of Malnutrition
Risks among Pediatric Surgical Patients. Balkan Med J 2014; 31(4):313-21.

34. Spagnuolo MI, Liguoro I, Chiatto F, Mambretti D, Guarino A. Application of a score system to evaluate the risk of malnutrition in a multiple hospital setting. Riv Ital Ped 2013; 39:81-88.

35. Joosten KFM, Hulst JM. Nutritional screening tools for hospitalized children: Methodological considerations. Clin Nutr 2014; 33(1):1-5.

36. Monte $\mathrm{CMG}$. Desnutrição: um desafio secular à nutrição infantil. J Ped 2000; 76(3):285-297.

37. World Health Organization. Physical status: the use and interpretation of anthropometry. Geneva; 1995. (Report of a WHO Expert Committee. Technical report series, 854).

\section{Como citar este artigo:}

Bousquet LA, Stringhini MLF, Mortoza AS. Avaliação nutricional subjetiva global: instrumentos para triagem em crianças hospitalizadas. Rev. Aten. Saúde. 2016;14(47): 67-74. 